\title{
Transformation of Chronic Myeloid Leukemia to Acute Biphenotypic Leukemia
}

\author{
Stanislav Ivanova, b, Purva Sharma ${ }^{a}$, Yash Jobanputra ${ }^{a}$, Yuanming Zhang ${ }^{a}$
}

\begin{abstract}
Chronic myeloid leukemia (CML) is a myeloproliferative disorder with clonal proliferation of all myeloid cell lines. The disease typically manifests in three phases: chronic course followed by an accelerated phase and finally a terminal blast crisis. A blast crisis is defined as the presence of $>20 \%$ blasts in the peripheral blood or bone marrow. The blasts could be characterized as either myeloid (60$80 \%$ of cases) resulting in acute myeloblastic leukemia or lymphoid (20-30\% of cases) resulting in acute lymphoblastic leukemia. In rare instances, a blast crisis could present with biphenotypic expression for both myeloid and lymphoid blasts. In such cases, about $6-10 \%$ of the time, the course of the disease is more aggressive and renders a poorer prognosis especially if there is evidence of extramedullary involvement. We present a case of a 41-year-old woman with history of CML who presented with acute biphenotypic blast crisis with extramedullary involvement in the context of aggressive chemotherapy. Literature review reveals $<10$ reported cases of CML with biphasic transformation and only three cases of CML with acute leukemia on presentation. Most cases described are in pediatric patients with varied presentations and none involving extramedullary sites. Mortality rates in such cases are near $100 \%$ despite aggressive chemotherapy.
\end{abstract}

Keywords: CML; Biphenotypic leukemia; Blast crisis; Extramedullary involvement

\section{Introduction}

Chronic myeloid leukemia (CML) is a myeloproliferative neoplasm characterized by the dysregulated production and uncontrolled proliferation of mature and maturing granulocytes with fairly normal differentiation. CML accounts for about

Manuscript submitted May 28, 2020, accepted June 17, 2020

Published online July 21, 2020

aUniversity of Miami/JFK Medical Center GME Consortium Internal Medicine Residency Program, Atlantis, FL 33467, USA

${ }^{b}$ Corresponding Author: Stanislav Ivanov, University of Miami/JFK Medical Center GME Consortium Internal Medicine Residency Program, Atlantis, FL 33467, USA. Email: s.ivanov1@med.miami.edu

doi: https://doi.org/10.14740/jmc3511
$15 \%$ of adult leukemia cases with slight predominance observed in men $[1,2]$. The median age at presentation is about 50 years of age. The disease manifests in three phases: chronic phase, accelerated phase and blast crisis. Presentation of biphasic transition of chronic leukemia directly to blast crisis is seen in aggressive disease courses and is associated with poorer response to treatment and worst outcomes. We present to you a case of a 41-year-old lady with CML who presented with diffuse adenopathy and was found to have biphenotypic presentation of leukemia.

\section{Case Report}

A pleasant 41-year-old woman presented to the emergency department with complaints of generalized body aches, fever, night sweats, and axillary adenopathy. The patient has known history of CML diagnosed 6 years prior to presentation and was initiated on imatinib treatment with good initial response to treatment but poor compliance thereafter. She was doing well until a week prior to presentation, when she developed fatigue and noticed swelling in her left axillary region. At the time of presentation, patient had stable vital signs. Physical examination was only remarkable for diffuse adenopathy. Remainder of the physical examination including cardiac and pulmonary systems was unremarkable. Laboratory studies revealed elevated white blood cell count of $30.4 \mathrm{~g} /$ dL with lymphocytic predominance $(48.8 \%)$. Pathologic review of peripheral smear was remarkable for leukocytosis with predominance of blasts. Bone marrow aspirate demonstrated profound hypercellularity with lymphoblasts $>70 \%$ consistent with lymphoid blastic phase. Immunohistochemical markers were positive for CD34, CD79a, PAX5 and TdT. Axillary lymph node biopsy demonstrated morphologic, flow cytometry and immunohistochemical findings consistent with CD34-positive blasts which were weakly positive for myeloperoxidase and CD7 and negative for PAX5 expression consistent with myeloblasts (Fig. 1). Chromosomal analysis revealed presence of BCR-ABL1 fusion, without mutation in the kinase domain, with additional expressions of MYC gene and notable trisomy of chromosome 8 and MLL gene rearrangement. Given the history, patient's presentation and pathology findings, the patient was determined to have CML with acute biphenotypic blast crisis transformation with extramedullary involvement. Salvage therapy was initiated with hyperfractionated cyclophosphamide, vincristine, adria- 


\section{Bone Marrow Specimen}
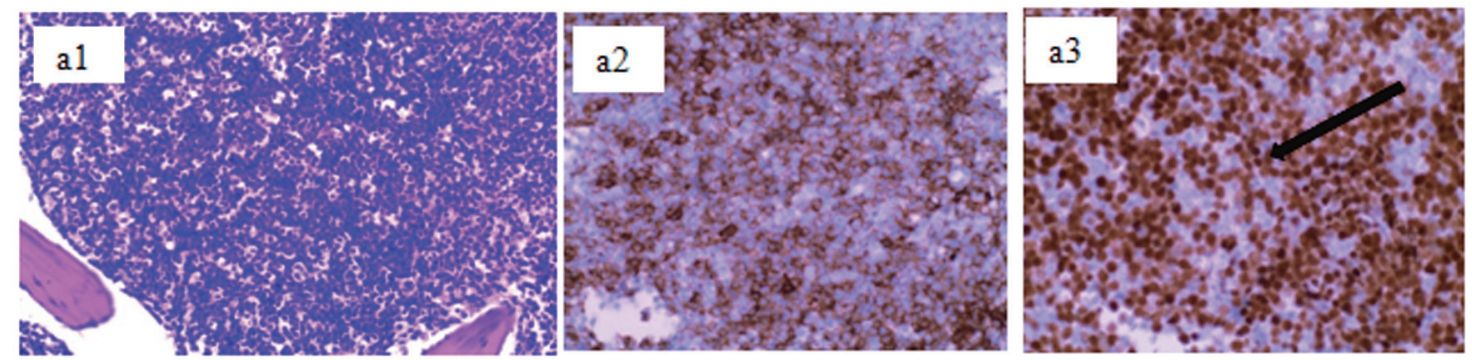

Lymph Node Specimen
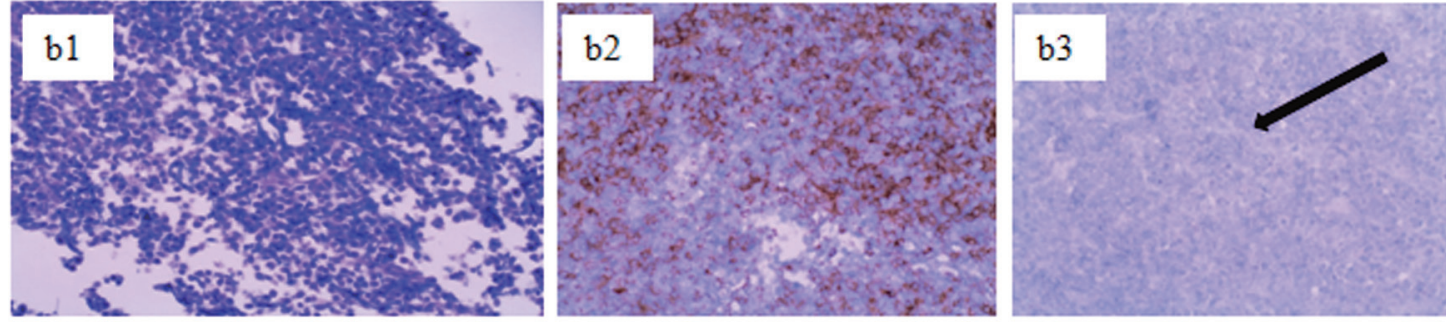

Figure 1. Pathology immunohistochemical staining of bone marrow (a) and lymph node (b) demonstrating increased expression of PAX5 in the marrow sample significant for B-cell blast $\left(C D 34^{+}\right)$population versus no expression of PAX5 in lymph node sample despite presence of blast cells $\left(\mathrm{CD} 34^{+}\right)$suggesting biphenotypic expression of the cell lines. Row a1: bone marrow, a2: bone marrow with CD34-positive expression, a3: bone marrow with significant expression of PAX5; Row b1: lymph node, b2: lymph node with positive CD34 expression, b3: lymph node without expression of PAX5.

mycin, and dexamethasone (hyper-CVAD ${ }^{+}$) imatinib regimen and intrathecal methotrexate and cytarabine. First cycle noted improvement in counts with acute decline from $107,000 / \mu \mathrm{L}$ to $60,000 / \mu \mathrm{L}$. Evaluation for allogenic hematopoietic stem cell transplant was initiated. A second cycle of the above regimen did not show signs of cytological response. The patient subsequently developed a subdural hemorrhage in the context of thrombocytopenia and expired during the course of the week.

\section{Discussion}

CML is a disorder of hematopoietic stem cells characterized by uncontrolled proliferation of mature and maturating granulocytes. Exposure to ionizing radiation is the only known risk factor [3]. The pathogenesis of the disease is associated with the fusion of two genes: BCR on chromosome 22 and ABL1 on chromosome 9 . This translocation, $\mathrm{t}(9 ; 22)(\mathrm{q} 34 ; \mathrm{q} 11)$, produces an abnormal chromosome 22 referred to as the Philadelphia $(\mathrm{Ph})$ chromosome which harbors the fusion gene, BCRABL1. The dysregulated, continuously activated tyrosine kinase protein product of this chromosome is implicated in the pathogenesis of symptoms $[3,4]$.

CML has a triphasic clinical course: a chronic phase, which is present at the time of diagnosis in approximately $85 \%$ of patients; an accelerated phase, in which neutrophil differentiation becomes progressively impaired and leukocyte counts are more difficult to control with treatment; and blast crisis, a condition resembling acute leukemia in which myeloid or lymphoid blasts proliferate in an uncontrolled manner [3]. The usual presentation is in the chronic phase. Blast crisis usually occurs in bone marrow; about $10-16 \%$ cases present with extramedullary involvement at the time of disease transformation from chronic phase. Common extramedullary sites include lymph nodes, bones, skin, testis, and nervous system. Presence of disease in such sites is associated with a significantly poorer response to therapy and survival [3].

Acute biphenotypic leukemia (ABL) is rare and is mostly seen in the pediatric population [5]. The incidence of ABL in adults ranges from $1 \%$ to $20 \%$ of acute leukemias [6]. There are no set diagnostic criteria for ABL. The European Group for Immunological Classification of Leukemias (EGIL) proposed a set of diagnostic criteria for ABL [7].

Studies of ABL have suggested that these leukemias may arise by malignant transformation of a pluripotent stem cell. The finding of a significantly greater percentage of biphenotypic cases with $\mathrm{CD}^{+} 4^{+}$(a marker of immature blast cells) expression compared to non-biphenotypic cases, as seen in our patient, supports the concept that this transformation process occurs at a step close to the hematopoietic progenitor cell [8]. Furthermore, the $\mathrm{t}(9,22)(\mathrm{q} 34 ; \mathrm{q} 11)$ is the most common cytogenetic abnormality described in ABL [9].

The development of ABL, as was the case here, is believed to be derived from marrow stem cells which have the capacity to express antigens from more than one cell line. These cells have notable immunohistochemical fingerprint with notable expression of CD79a, which serves as transmembrane signal modulator early during the course of B-cell differentiation. Other common associations for the development of ABL in- 
clude the increased expression of MLL gene product (myeloid/lymphoid leukemia) which plays a key role in the establishment of progenitor cells during hematopoiesis. The MLL gene product itself has two unique characteristics in that the N-terminal of MLL protein product forms a fusion protein with the C-terminal of over 40 different partners, including itself, and MLL rearrangements are found in both ALL and AML, whereas most other translocations are lineage specific thus allowing for dual lineage proliferation and development of leukemic symptoms seen in both AML and ALL [10]. Such findings were seen in our patient who presented with profound anemia and thrombocytopenia, leukocytosis, fever and night sweats as well as invasion of extramedullary tissue [11, 12].

Other factors implicated in the pathogenesis of $\mathrm{ABL}$ are compliance to therapy. The Adherence Assessment with Glivec: Indicators and Outcomes (ADAGIO) study has shown that non-adherence to imatinib therapy is shown to have higher correlation with poorer outcomes, likely secondary to accumulation of somatic mutation leading to resistance and thus subsequent transformation from a chronic state to an accelerated phase and subsequently a terminal blast crisis [13]. As seen with this patient, interruption of imatinib treatment contributed to the development of far more aggressive disease course characterized by biphenotypic expression and extramedullary involvement [14].

Treatment of ABL is challenging and the optimal therapy for this sub-type of leukemia remains unclear. A case series by $\mathrm{Xu}$ et al showed that the relapse rate in ABL patients was significantly higher than that in AML patients, while the complete remission (CR) rate after relapse was lower than that in AML patients. Also observed was low CR rate after relapse in ABL patients when compared with ALL patients [15]. Allogenic hematopoietic stem cell transplantation (HSCT) is an effective treatment for ABL patients, regardless of the existence of any known prognostic factors, with the exception of nonremission (NR) as long as the disease is sufficiently controlled until HSCT [16].

Management of CML with acute transformation to $\mathrm{ABL}$ with blast crisis is a widely debated topic in the hematology/ oncology realm as no clear regimen has been shown to have definitive benefit in decreasing the morbidity and mortality of the disease. Selection of ALL specific versus AML specific protocol for treatment has yet to be explored. Studies are currently being conducted to select the best course regimen with focus on HSCT as treatment modality; however, the aggressive nature of the disease challenges designs of such study protocols [17].

\section{Conclusions}

The prognosis of ABL patients is poor when compared with de novo acute myeloid leukemia or acute lymphoblastic leukemia. ABL patients showed a much higher incidence of CD34 antigen expression, complex abnormal karyotype, extramedullary infiltration, relapse, and resistance to therapy after relapse. Further studies are needed to determine the prognostic factors of $\mathrm{ABL}$ and treatment modalities.

\section{Acknowledgments}

We would like to thank JFK Medical Center in Atlantis, FL, USA.

\section{Financial Disclosure}

None to declare.

\section{Conflict of Interest}

None to declare.

\section{Informed Consent}

Not applicable.

\section{Author Contributions}

SI collected data and prepared draft of abstract, case presentation, discussion and conclusion. YZ evaluated and prepared pathology reports from bone marrow and lymph node specimens. PS was the final draft reviewer. YJ was the final draft reviewer.

\section{Data Availability}

The authors declare that data supporting the findings of this study are available within the article.

\section{References}

1. Siegel RL, Miller KD, Jemal A. Cancer Statistics, 2017. CA Cancer J Clin. 2017;67(1):7-30.

2. Chen Y, Wang H, Kantarjian H, Cortes J. Trends in chronic myeloid leukemia incidence and survival in the United States from 1975 to 2009. Leuk Lymphoma. 2013;54(7):1411-1417.

3. Faderl S, Talpaz M, Estrov Z, O'Brien S, Kurzrock R, Kantarjian HM. The biology of chronic myeloid leukemia. N Engl J Med. 1999;341(3):164-172.

4. Ariyasu T, Matsuo Y, Harashima A, Nakamura S, Takaba S, Tsubota T, Orita K. Establishment and characterization of "biphenotypic" acute leukemia cell lines with a variant $\mathrm{Ph}$ translocation $\mathrm{t}(9 ; 22 ; 10)(\mathrm{q} 34 ; \mathrm{q} 11 ; \mathrm{q} 22)$. Hum Cell. 1998;11(1):43-50.

5. Al-Seraihy AS, Owaidah TM, Ayas M, El-Solh H, AlMahr M, Al-Ahmari A, Belgaumi AF. Clinical characteristics and outcome of children with biphenotypic acute leukemia. Haematologica. 2009;94(12):1682-1690.

6. Hanson CA, Abaza M, Sheldon S, Ross CW, Schnitzer 
B, Stoolman LM. Acute biphenotypic leukaemia: immunophenotypic and cytogenetic analysis. Br J Haematol. 1993;84(1):49-60.

7. Bene MC, Castoldi G, Knapp W, Ludwig WD, Matutes E, Orfao A, van't Veer MB. Proposals for the immunological classification of acute leukemias. European Group for the Immunological Characterization of Leukemias (EGIL). Leukemia. 1995;9(10):1783-1786.

8. Legrand O, Perrot JY, Simonin G, Baudard M, Cadiou M, Blanc C, Ramond S, et al. Adult biphenotypic acute leukaemia: an entity with poor prognosis which is related to unfavourable cytogenetics and P-glycoprotein overexpression. Br J Haematol. 1998;100(1):147-155.

9. Hossain D, Weisberger J, Sreekantaiah C, Seiter K. Biphenotypic (mixed myeloid/T-cell) extramedullary myeloid cell tumor. Leuk Lymphoma. 1999;33(3-4):399-402.

10. Muntean AG, Hess JL. The pathogenesis of mixed-lineage leukemia. Annu Rev Pathol. 2012;7:283-301.

11. Juneja R, Dhamija G, Dadu T, Handoo A. Biphenotypic extramedullary blast crisis with MLL gene rearrangement in a case of chronic myeloid leukemia following Dasatinib therapy: An unusual case. Indian J Pathol Microbiol. 2016;59(2):259-260.

12. Oh SH, Park TS, Kim HR, Lee JY, Kim JH, Shin JH, Lee JN. Chronic myelogenous leukemia showing bipheno- typic blast crisis followed by lineage switch to B lymphoblastic leukemia. Leuk Res. 2009;33(11):e195-198.

13. Noens L, van Lierde MA, De Bock R, Verhoef G, Zachee P, Berneman Z, Martiat P, et al. Prevalence, determinants, and outcomes of nonadherence to imatinib therapy in patients with chronic myeloid leukemia: the ADAGIO study. Blood. 2009;113(22):5401-5411.

14. Hochhaus A, Larson RA, Guilhot F, Radich JP, Branford $\mathrm{S}$, Hughes TP, Baccarani M, et al. Long-term outcomes of imatinib treatment for chronic myeloid leukemia. N Engl J Med. 2017;376(10):917-927.

15. Xu XQ, Wang JM, Lu SQ, Chen L, Yang JM, Zhang WP, Song XM, et al. Clinical and biological characteristics of adult biphenotypic acute leukemia in comparison with that of acute myeloid leukemia and acute lymphoblastic leukemia: a case series of a Chinese population. Haematologica. 2009;94(7):919-927.

16. Mori J, Ishiyama K, Yamaguchi T, Tanaka J, Uchida N, Kobayashi T, Fukuda T, et al. Outcomes of allogeneic hematopoietic cell transplantation in patients with biphenotypic acute leukemia. Ann Hematol. 2016;95(2):295-300.

17. Wadhwa J, Szydlo RM, Apperley JF, Chase A, Bua M, Marin D, Olavarria E, et al. Factors affecting duration of survival after onset of blastic transformation of chronic myeloid leukemia. Blood. 2002;99(7):2304-2309. 